\title{
Business and Industry Specific Cloud: Challenges and Opportunities
}

\begin{tabular}{|c|c|c|}
\hline Anne James & and & Jen-Yao Chung \\
\hline Distributed Systems and & & \\
\hline Modelling Research Group, & & Quanta Cloud \\
\hline Coventry University & & Technology \\
\hline Priory Stroet & & Tao Yuan Shien, \\
\hline Priory Street, & & Taiwan \\
\hline CV1 5FB & & 33377 \\
\hline a.james@coventry.ac.uk & & jy.chung@qcttw.com \\
\hline
\end{tabular}

This guest editorial highlights the current move by many cloud providers to business and industry -specific cloud. We summarise the latest happenings and provide examples of business and industry -specific needs by considering some case study reports and related research. We introduce four new special section papers, which further elaborate on issues and solutions relevant to business and industry -specific cloud. Finally we summarise the challenges and opportunities ahead for researchers and developers.

\section{Introduction}

Hailed as the next big thing $[1,2,3]$, industry-specific cloud or the development of cloud provision to meet the specific needs of a particular industry, is a recognised direction supported by recent IDC reports [4, 5]. In fact business and industry -specific cloud is seen as a logical progression for cloud and as required by the market-place if cloud is to grow. A number of big players are launching initiatives in this field. At the 2013 International Conference on E-business Engineering held at Coventry University UK (ICEBE 2013) the topic was aired amongst the delegates and for that reason we decided to create a special section on the subject in a relevant journal. We are delighted that the Future Generation Computer Systems journal has facilitated this. Whilst being an area that has attracted more industrial than academic attention at the moment, it is hoped that this special section will trigger responses from researchers that will help to further the developments in this field.

In this extended editorial, we describe the latest happenings in the cloud provider industry and we also discuss relevant research in the area, highlighting some case studies as well as 
research papers which address relevant aspects. We introduce the papers of the special section which comprise four selected, peer-reviewed papers relating to business and industry -specific cloud either by describing novel architectures for specific domains or by offering new solutions to improve cloud provision which are exploitable by the industryspecific environment. The special section papers are extensions of selected papers presented at ICEBE 2013. Finally in our editorial, we offer a view on the challenges and opportunities in this new direction of cloud provision.

\section{Happenings in Industry}

Prevailing cloud solutions do not fully address the specific needs of particular industrial sectors. Some of the reasons that can dissuade an enterprise of using a cloud-based solution are concerns regarding security, privacy, control and interoperability of data. The idea of putting all one's data in one place might not seem ideal and furthermore concerns about the appropriateness of the functionality provided can stop some users adopting Software as a Service (SaaS) solutions. For such reasons a new direction being followed by many big cloud providers is a move towards industry-specific, also sometimes called vertical, cloud where such issues can be addressed and where provision can be tailored specifically for the customer and its industry. The market research company IDC has predicted continued significant growth in cloud with a greater concentration on business solutions as opposed to platform and infrastructure over the next few years [5]. It is expected that industry expertise will increasingly be required to win cloud professional services contracts.

Aware of the changing perceptions and needs within the market, many big providers are moving towards vertical solutions. Industry sectors that have been addressed include: insurance; legal services; health; banking; manufacturing; education; human resources (HR); retail; transportation; government; media and entertainment; pharmaceuticals; and energy/utilities (see Figure 1). The needs of, say, the healthcare sector would obviously be quite different from that of the manufacturing sector, thus it makes sense to tailor provision. The differences can occur at various levels from infrastructure needs, growth patterns, software functionality, privacy and security to the requirement to interoperate with third parties. 


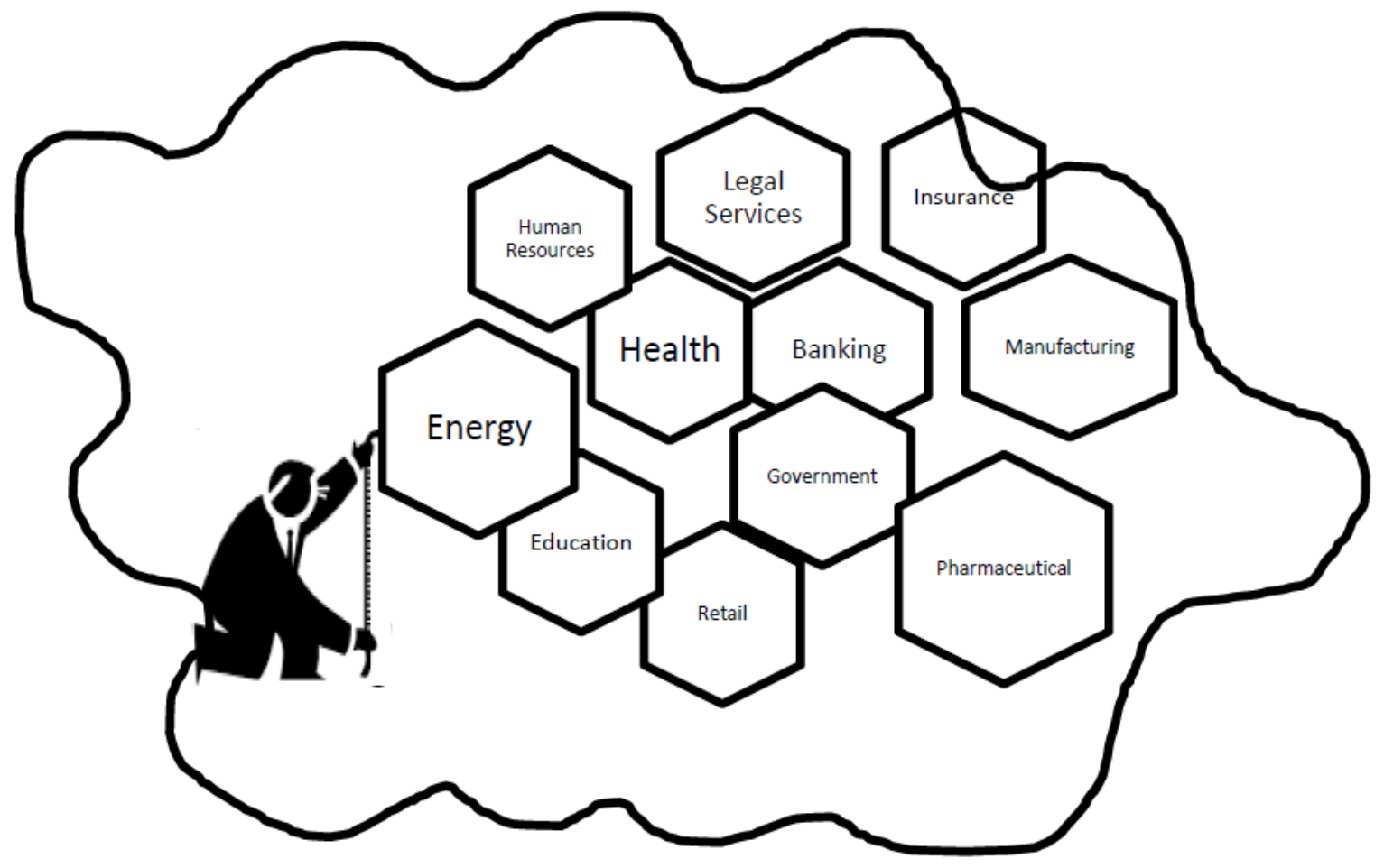

Figure 1: Tailored Cloud Services for various Sectors

When users move to a cloud environment, specific business functionality can be lost. Therefore clients need to carefully assess their needs when migrating. Warranting consideration are the questions of which areas will benefit most from a cloud-based service delivery model and whether any are better left in on-premises style of delivery. Workload analysis can aid in the process of such an assessment and the availability of choice between public, private or hybrid cloud can provide a solution for such varying needs. Recognising these requirements, major cloud providers are tailoring their solutions appropriately to support different types of cloud. The main method of providing industry-specific cloud is through services. The service model enables functionality to be delivered in stand-alone packages suitable for cloud delivery. However the functionality must currently exist as a service or would need to be redesigned as such. Alternatively suitable services, which deliver the right functionality, might be discovered from third parties. Cloud providers are addressing the functionality issue by providing ready-made standard software services to deliver specific functionality through services. 
IBM recently created 12 industry-specific subscription-based cloud solutions [6, 7]. Examples include the IBM Insurance Service Hub Cloud which enables interaction between clients, health insurance companies and multiple data providers such as clinics and physicians through a standardised interface. IBM have also developed a Human Capital Management tool which enables HR to move from an on-premises solution to a cloud environment which provides analytics, self-service talent management as well as compensation and succession planning. The company also provides a catalogue of rolebased SaaS applications to enable enterprise leaders to drive their business areas to better results (C-Suite). Other releases include: specific healthcare; mobility; asset management; customer data; and predictive asset management tools.

Based on its extensive experience of various industries, the company, SAP, plans to direct more of its revenues into growing the market share of its SaaS provision and SAP Business Suite [8]. It has recently launched an Industry Cloud unit to address arising industry-specific concerns with cloud-based solutions. SAP intends to work with customers to develop prescriptive industry-specific road maps and cloud solutions for 25 industries. Deployable across public, private, or hybrid clouds, the provision will include intuitive interfaces and processes to simplify business. It will also include accessible big data.

Industry-specific virtualized desktop solutions for education, government, financial services, healthcare and manufacturing clients have been launched by VMWare [9]. Various cloudbased functionalities have been provided suited to the specific industry. For example: the education-based tool offers facilities for delivering tests and storing results; the government tool provides simplified and more advanced data protection technology; the financial services tool enables workers to use mobile devices securely; the healthcare solution enables monitoring of healthcare networks to ensure better quality of service; and the manufacturing tool offers 3-D graphics.

Smaller players are also active in the industry-specific market. Partnering with experienced valued added resellers already established in the legal industry, dinCloud have been gearing to the demands of the information-centric legal services [10]. A hosted virtual desktop, which can be run turnkey alongside a virtual private data centre, addresses the statutory need for redundancy and resiliency in this field as well as meeting the requirement for virtual office functionality in order for legal staff be available quickly and conveniently to clients. Through its library of 150 diverse SaaS applications, Cetrom, another cloud computing provider, supplies solutions and technology services to meet the needs of specific industries 
[11]. Application examples include customer relationship management (CRM), graphic design and mobile workforce support. Cetrom offers custom programming if the required application is not available. Cloud platform provider Veeva has tackled the requirements of the life sciences industry through a redesign of the Salesforce.com template [3]. Veeva's life sciences CRM provides for drug sample tracking with electronic signature capture, healthcare affiliations management, and interactive, rich media demonstrations. Its Vault service allows for the secure storage of documents such as those from for the clinical trials processes and supports versioning and approvals. Through Veeva's Network service it is possible to maintain a healthcare provider master data set.

We can see from the above paragraphs that major cloud providers are making notable effort to develop industry-specific offerings. Examples have been given of the different types of needs of diverse industries. Whilst most of the industry efforts so far have been in SaaS layer, there is space also in other layers to tailor provision to specific industry. Figure 2 provides our view on how cloud services have been developing.

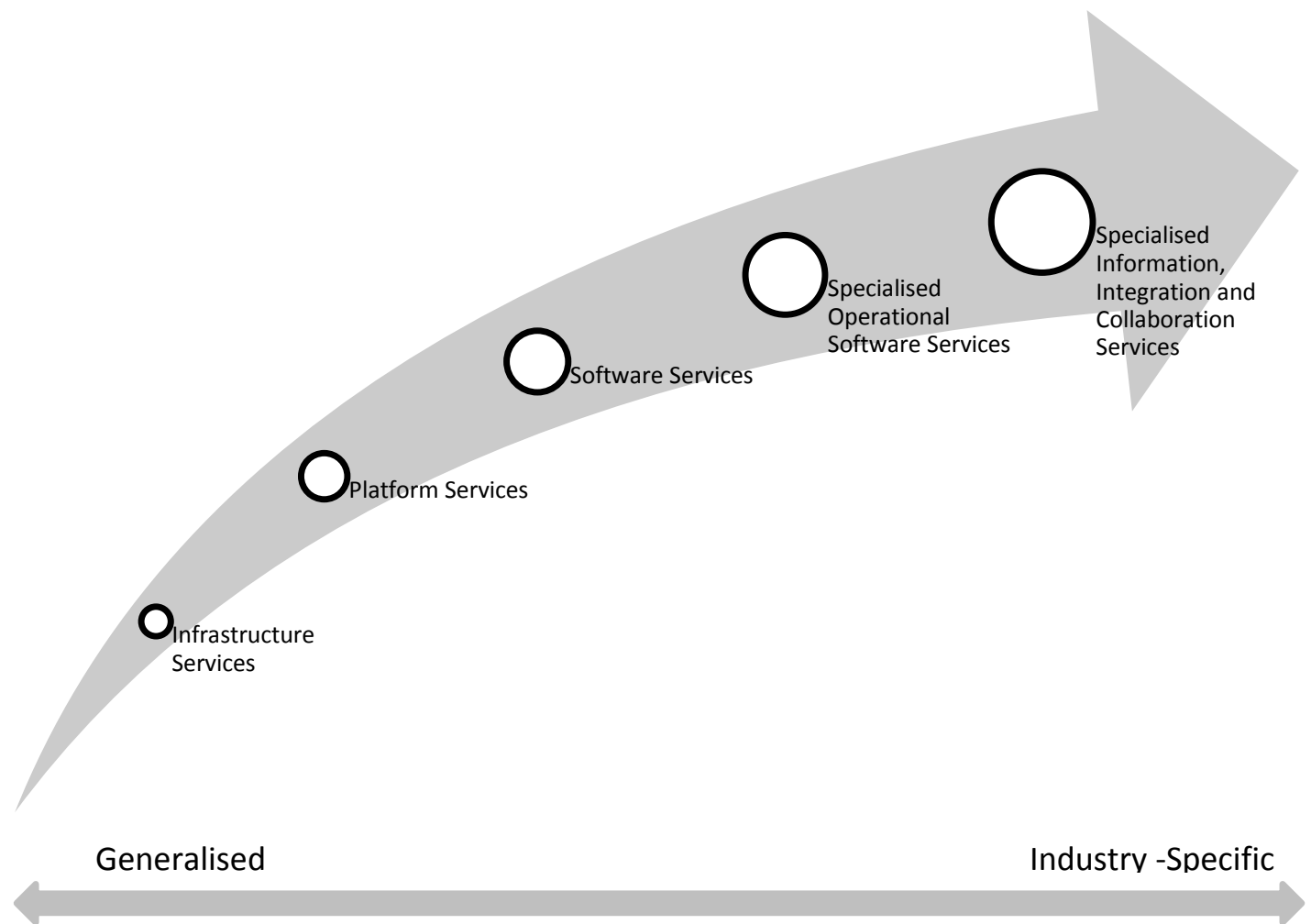

Figure 2: Development of Cloud Services towards Business and Industry Specific Provision 


\section{User Case Studies}

Some case studies have been published in the recent literature about domain-focussed applications being fully or partially moved to cloud. The areas of environment, health, energy, finance, and manufacturing have recently received attention. The following paragraphs consider some of the findings.

In 2013 Sun described the migration of an environmental decision support systems (EDSS) module from the traditional client-server-based architecture to a client of cloud-computing services [12]. This particular EDSS was concerned with watershed management which involves consideration of water supply, water quality, drainage, storm water runoff, water rights and overall planning. According to the authors watershed management is essentially a participatory process, requiring collaborations among multiple institutions and stakeholders. Data sharing and transparency is very important. An EDSS typically consists of multiple environmental models, databases, and assessment tools integrated under a graphical user interface, often realized through geographical information systems (GIS) [13]. The authors remark that a watershed EDSS can include dozens of GIS layers, ranging from coarse-scale geopolitical maps to fine-resolution land-use/land cover (LULC) maps. This means that a traditional client-server approach often does not provide the performance that users may expect given to what they may have become accustomed with other common types of system. For this reason the author sought to explore a cloud computing solution to provide additional resource in the running of a watershed EDSS. Google Drive, with the underlying Google Fusion Table, was used to provide basic visual analytics features that can be used to increase the collaborative decision-making experience while drastically reducing the cost. The results were favourable, with collaborative decision-making through cloud implementation able to be supported at a fractional cost of a full-scale, client-server application. However the author recognised that more sophisticated EDSS could be achieved through further leverage of combined client-server and cloud architectures and provision of additional cloud-based apps for environmental management. This case study highlighted the need for further investigation into specific architectures and development of specialised services for specific industries. 
Health information systems (HIS) were considered by Poppe et al. in 2013 [14] who described the use of cloud computing for health information in Ghana and Kenya. The cases presented demonstrated how a web-based, online architecture for HIS could be advantageous over the standalone, offline systems. Of particular mention was the benefit of being able to compare data across different services. However it was noted that attention should be paid to other aspects. These included skills development, policies for system administration and consideration of system ownership. The lessons to be drawn for cloud providers are that clients are often required by law or perceptions of ownership responsibility to hold data within national boundaries and to exercise some control over it. Such facilities must be offered to allow localised storage and also services should be supplied to allow users to administer their system. For similar reasons providers need to consider offering training in system administration so that customers can use services that access lower levels of the system stack. Thus the contractual boundaries between Infrastructure as a Service (laaS), Platform as a Service (PaaS) and Software as a Service (SaaS) may be to be redrawn for specific applications and industries. We can understand from these case studies the importance of integration and data comparison. Providers addressing verticals should therefore include in their offering suitable services to allow companies to integrate and compare data in ways suitable to their business or industrial domain.

Perrons and Hems in 2013 [15] discussed cloud provision in the upstream oil and gas industry. This industry faces several technical challenges including an emphasis on data security, a reliance on extremely large data sets, and significant legacy investments in information technology infrastructure. Currently the authors consider a hybrid solution to be the most appropriate method given these challenges. A hybrid solution consists of combined private and public cloud. However the authors consider that with the emergence of more suitable cloud-based apps for the industry, further momentum towards public cloud could be achieved. We can glean for this work that there is a need for more specific services towards large data set handling and security to further advance the cloud approach in the energy field. Energy was also addressed by Dan et al. who turned their attention to the power grid [16]. The authors noted that electric power utilities rely heavily on communications and computation to plan, operate and analyze power systems but adoption of cloud facilities has been slow, chiefly because of security concerns. The authors argue that providing security 
properties through both application layer composition and via assured cloud computing would enable further take-up.

An assessment of the relevance of data privacy requirements of financial institutions in the context of cloud computing was made by Wenge at al. in 2014 [17]. Following a survey of stakeholders in a major financial institution, their results indicated that certain data privacy issues still form obstacles for the adoption of cloud computing even when administrative countermeasures and legal agreements are in place.

In 2013 Wang and Xu [18] addressed cloud manufacturing (CManufacturing) which is a more complex concept than cloud computing. It is not simply a migration of manufacturing software to the cloud. It involves sharing and communication across various organisations that might form part of the manufacturing supply chain. CManufacturing has been described as a service-oriented IT environment which forms a basis for the next level of manufacturing networks by enabling production-related inter-enterprise integration down to shop floor level [19]. Manufacturing enterprises and related capability need to be described, componentised, virtualised and integrated in a Mcloud. Wang and $\mathrm{Xu}$ assess that there is not yet a cloud solution for the entire manufacturing supply chain. Lacking is a supervision mechanism to organize and control services as well as facilities to integrate current and future manufacturing resources. Following a review of other work, Wang and Xu develop standardized data models describing cloud services and relevant features for manufacturing. These include services for product design, simulation, CNC milling, precision welding and grinding. The authors conclude that cloud technology brings benefits such as openness, cost-efficiency, resource sharing and production scalability. However CManufacturing faces a tougher challenge than just implementing manufacturing-related software in the cloud. Unlike software programs and IT infrastructure, physical machines, monitors, and facilities cannot be easily deployed on the cloud, although theoretically at its most advanced level, and with careful scheduling, services could be taken right down to the level of controlling physical machines. To implement CManufacturing the intermediate processes from raw material to finished products must be well understood. This niche area can be a challenge for cloud providers. In particular, interoperability must be a foremost concern because of the nature of the supply chain requiring interaction between separate enterprises. The area of 
computer supported cooperative work in design has been growing and has been recognised as one for which better solutions for interoperability and integration are required [ 20, 21, 22]. The growth in interactions between companies in manufacturing demands appropriate specific solutions for interoperability which will be found through closer collaboration between service providers and domain experts.

Although not specifically addressing a vertical, it is interesting to consider the work of Gupta, Seetharaman, and Raj [23]. These authors investigated the appropriateness of cloud technology for small and medium enterprises (SMEs). They found that this group did not consider the cloud to be reliable and preferred their conventional methods for sharing and collaborating with their stakeholders rather than using cloud for sharing and collaboration. However this group liked ease of use and convenience of the cloud and also security and privacy and the cost reduction. It is interesting that this group were satisfied with the security and privacy afforded by the cloud, an area with which many verticals have concern. In this case, it seems that SMEs are more confident in the delivery of security and privacy through a utility computing paradigm than through in-house efforts. SMEs were also addressed by Li et al. [24] who consider that no cloud computing service vendor can satisfy the full functional information system (IS) requirements of an enterprise. Thus companies typically use both cloud and intra-enterprise ISs. The authors state that given that the operation logic of the cloud can be considered a 'black box' for users, integration of the enterprise business logic with the logic of the cloud will be difficult.These authors go on to present a framework to integrate applications deployed in public clouds and intra-enterprise ISs and which allow cross-communication between the two architectures. Based on business process engineering, key aspects of the framework are collaboration agents (CAs) and collaboration points (CPs). The CPs bring together APIs of the cloud providers and the intra-enterprise ISs, encapsulated as web services. The CAs hold the business logic and realise the service orchestration necessary at the CPs. An application service integration framework was developed together with an application services integrating platform. This research highlights the current tendency of SMEs to use a mixture of various cloud provider services and inhouse services to satisfy their computing requirements. With regard to the move towards industry-specific services by cloud providers, it is likely that these issues of interoperability will lessen as providers supply increasing numbers of relevant services to enterprises. 
However the issue of portability remains and also the issue interoperability would remain relevant for inter-company collaboration in cases where collaborating companies use different cloud providers.

Interoperability is in fact an area of prevalent concern. A number of researchers have given attention to this issue $[25,26,27,28,29]$. There is a fear of vendor lock-in with moving to the cloud. This could occur if provider software does not have suitable Application Program Interfaces (APIs) that can allow interaction across organisations or easy migration to new providers. This problem can affect some industries more than others. Those heavily dependent upon supply chain or inter-organisational interaction will be less inclined to move to the cloud if it is going to hinder interaction. Petcu et al. [27] note that open APIs, standards and protocols with early integration in the software stack are the key elements towards a solution to this problem. These authors discuss an approach for a new set of APIs for cloud application development from the point of view of portability. For the cloud to move forward in the industry-specific field, open APIs are required. Given these, vendors can provide suitable integrative services if they understand well the ways in which companies interact in a specific field. Mezgár and Rauschecker [28] note that the issues of handling interoperability, portability and standardization challenges have not been solved fully yet. Addressing the manufacturing sector, they consider that there are different types of networked enterprise. An overview is given on interoperability and standardization issues in cloud computing environments. Furthermore a taxonomy on possible connecting forms of networked enterprises and cloud-based IT systems is provided.

Security and privacy are other common concerns. Some industries have legal requirements regarding handling of data. Companies are also are bound by government requirements. In many cases this means that data must stay within national boundaries. Furthermore companies do not like to relinquish control to third party providers, particularly if they do not reside within the same national boundaries, a situation which could give rise to questions of applicable jurisdiction. Marsten et al. 2011 [29] provide a comprehensive analysis of these issues.

The idea of Information as a Service was explored by Demirikan and Delen [30]. This concept revolves around the idea of services that will efficiently integrate available information about a particular entity. We can view this type of service as sitting at a higher level than operational software services. For example, Ma, Li and Zhou [31] have proposed a dedicated creditworthiness service which collects online and professional comments about 
of products, and calculates credentials for ability or quality. In the era of big data, collection, integration and analytical services tailored to particular business and industry needs is most valuable and the area offers good opportunity for development by industry-specific cloud providers and researchers.

From the case studies we can conclude that security, privacy, control and interoperability remain concerns for industries as well as the need for more specialised services at application level. If more specialised services both for applications and system administration based on greater domain knowledge could be made available, cloud take-up would increase.

\section{Special Section Papers}

This special section contains four papers addressing aspects relevant to industry-specific cloud. Two of the papers describe application areas which have adopted a particular and novel form of cloud suitable to the specific domain. The other two cover more general techniques which have significance for vertical cloud in that their application can be made even more effective with the extra knowledge that would be available through business and industry -specific understanding.

The first paper by Chao et al. [32] describes results of a European Union (EU) project, namely the Cloud E-learning for Mechatronics (CLEM) project. CLEM is an example of a domain-specific cloud that is especially tuned to the needs of VET (Vocational, Education and Training) teachers. A specific cloud platform has been developed which provides static and dynamic resources. The static resources take the form of a traditional e-learning system, except that it is delivered through the cloud. The dynamic part consists of remote laboratories that can be accessed and used through the cloud. A further novelty in the work is the ecosystem framework which enables the static and dynamic resources to grow organically in a Web 2.0 fashion. Additionally facilities are provided for tailored collaborative interaction among users and experts. An aim of this project was to provide an educational tool which would promote the growth of the skills base in the field of mechatronics across the EU. It is an important example of a new type of cloud for a specific domain.

Kumar et al. elaborate on the Vehicular Mobile Cloud in our second special section paper [33]. The vehicular mobile cloud enables vehicular users to access various resources from 
the cloud which acts as a service provider for them. However transmission in this type of system is not always reliable due to the dynamic nature of the environment characterized by moving vehicles. The authors formulate the problem of reliable data forwarding as a Bayesian Coalition Game (BCG) using Learning Automata concepts. Learning automata are stationed on the vehicles and assumed to be players in the game in which rewards or penalties are given from the environment depending on decisions taken. The results obtained show that the proposed scheme is better than the other conventional schemes with respect to metrics such as message overhead, throughput and delay. The proposed architecture is an example of a hybrid cloud where some processing and data is realised through a cloud facility whereas other is accomplished through peer-to-peer communication as typical of the application domain. Providers in this area would need to supply specialized central services whilst facilitating integration with peer-to-peer communication methods in the form of ad hoc networks.

$\mathrm{Ma}$, lan and $\mathrm{Li}$ [34] provide the third paper which tackles the issue of discovering services which fit into the user's particular context. The authors propose a Contextual Service Discovery (CSD) approach to help find qualified services in accordance with binding context on the user side. Two main advantages of the approach are highlighted: firstly that queries are expanded to minimize the mismatch between terms that are lexically or semantically related; and secondly that the contextual features of service interface compatibility is considered rather than only the textual and semantic similarities. The discovery works by first lexical matching and then matching on binding context. The composition of services in a flow is important for context and matching requires checking that output from one service in the flow is compatible with the required input for the next service in the flow. In the context of industry-specific cloud, this paper highlights the challenges of discovering the right services for particular areas of application. In industry-specific cloud it is likely that much of this work will have been done by the vendor following careful analysis of the needs of the industry. However as noted in section 3, interoperability between clouds is likely to be a prevailing issue since collaborating enterprises might adopt disparate cloud providers. Furthermore within the vendor domain, alternative services that fulfil similar functions may be available. In this context the type of discovery system described in this paper could be very useful. Large service vendors need to be able to adapt quickly to changing needs of particular industries and thus may need to quickly find appropriate services to satisfy needs. A discovery system of this sort could be useful to vendors who may need to customize services for a particular client or introduce a new service to satisfy an identified need. 
The issue of energy consumption in data centres is the subject of the fourth and final paper in this special section. Shen et al. [35] note that much of that energy is wasted maintaining excess service capacity during periods of low load. To solve this problem the authors investigate the problem of "rightsizing" the data centre for energy-efficiency through virtualization which allows consolidation of workloads onto a smaller number of servers while dynamically powering off the idle ones. However the tradeoff between energy saving and performance is highlighted. A stochastic model based on Queueing theory is proposed to capture the main characteristics of the problem. To mitigate the tradeoff between the energy conservation and performance, the authors develop an algorithm which searches for the optimal system parameter values for "right-sizing" such that energy is saved whilst performance is maintained. The authors present the Stochastic Right-sizing Model (SRM) and report on test results which show that SRM can significantly reduce energy consumption while keeping high performance. Let us consider the significance of this research to the move towards industry-specific cloud. A problem facing systems that aim to dynamically conserve energy is the lack of knowledge of up-coming needs. Switching servers off for a short period only to switch them on again when more tasks appear could be inefficient in terms of energy consumption and equipment life expectancy. Therefore accurate predictive modelling is important and this is recognized by the authors of the paper. Industry-specific cloud could be exploited to help in this area. The introduction of industry-specific cloud would enable more accurate prediction of processing needs as greater understanding of the underlying domain creates better knowledge. Furthermore services tailored to a specific industry are likely to be more efficient than general-purpose services.

The special section papers have highlighted the need for different types of cloud architecture to service different industries as well prompting us to consider how services for discovery and energy saving can gain extra potential through industry-specific knowledge.

\section{Challenges and Opportunities}

Given the findings from our review in the previous sections, we can conclude that in order for cloud providers to gain momentum in the industry-specific market, the following features are required of their provision:

- suitable specific functionality at the application level,

- higher level data collection, integration and analytical services that range across platforms and domains as necessary, 
- robust security and privacy services tailored to the specific industry at application, and platform levels,

- tailored platforms,

- hybrid solutions adapted to the specific industry for companies that will not move fully to cloud,

- varying levels of system administration services,

- choice of data store location so that data can remain in-country,

- solutions to enable interoperability so that clients are not locked-in,

- new architectures for specialised environments,

- industry-specific energy consideration,

- training provision according to need,

- $\quad$ suitably adapted policies for ownership and responsibility.

Requiring close interaction with domain experts, the above areas offer challenges and opportunities for both researchers and vendors at various levels of the cloud service stack (see Figure 3).

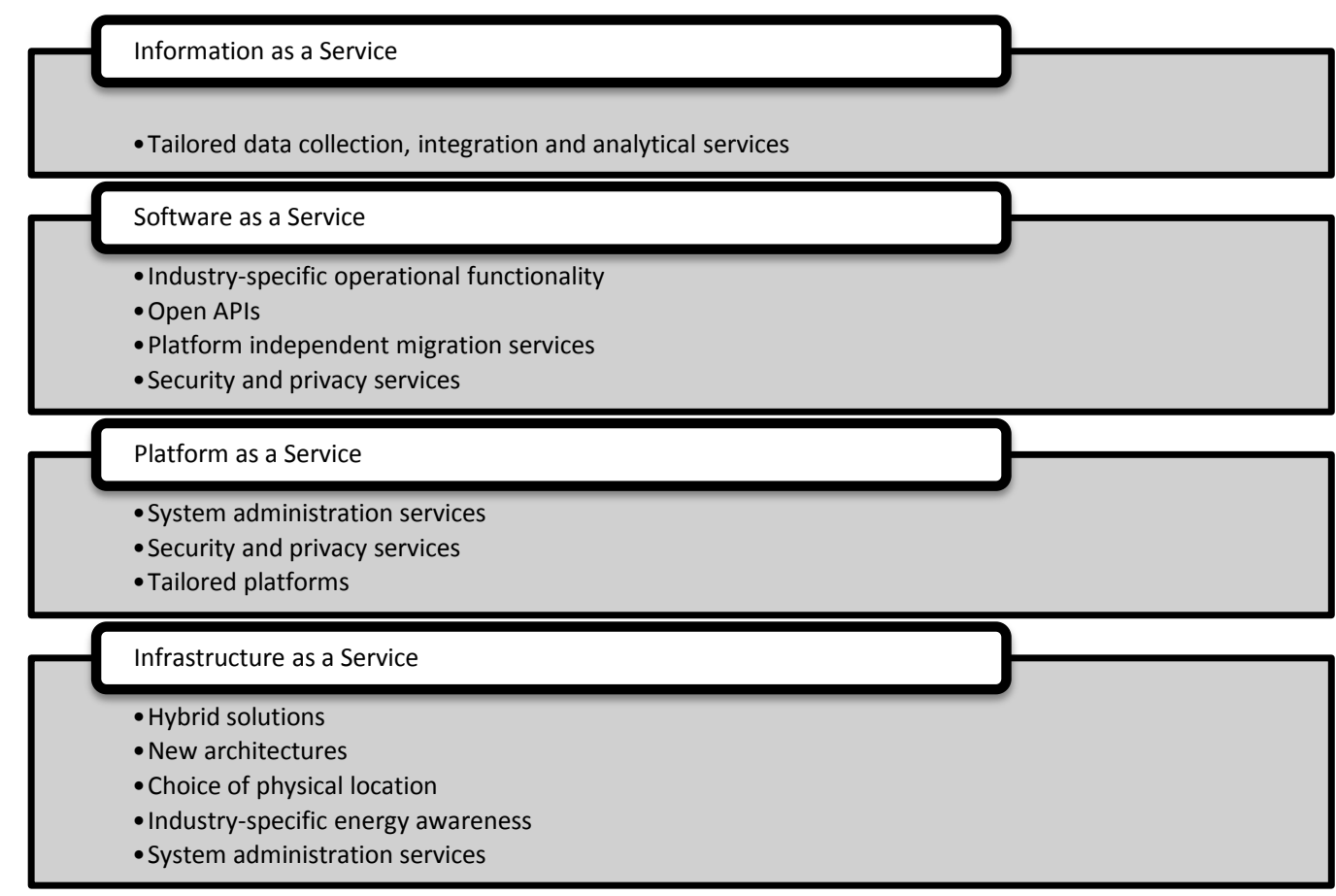

Figure 3: Challenges and Opportunities in Business and Industry Specific Cloud 


\section{Conclusion}

A number of major cloud providers are turning their attention to industry-specific cloud which provides specialised services for particular vertical domains. Security, privacy, control and interoperability remain concerns for industries as well as the need for more specialised services at application level. Individualised cloud architectures need to be provided as well as specialised software to meet disparate needs. In the future, cloud providers will need to integrate more closely with domain experts if cloud is to continue to grow and overcome common concerns.

\section{References}

[1] CloudWedge, The Rise of Industry Specific Clouds, available at : http://www.cloudwedge.com/rise-industry-specific-clouds/_ [accessed 12/11/2014]

[2] A. Baig, 2014 Cloud Trends Outlook - Future of Cloud Services, available at : http://talkincloud.com/cloud-computing/032114/2014-cloud-trends-outlook-future-cloud-services [accessed 12/11/2014]

[3] J. Kaplan, The Cloud's Long Tail: Industry-Specific SaaS Solutions, available at: http://www.ecommercetimes.com/story/80281.html [accessed 12/11/2014]

[4] G. Little, C. Huston and R.Segal , IDC MarketScape: Worldwide Cloud Professional Services 2013 Vendor Analysis, Doc \# 242401

[5] IDC Press Release, IDC Forecasts Worldwide Public IT Cloud Services Spending to Reach Nearly $\$ 108$ Billion by 2017 as Focus Shifts from Savings to Innovation http://www.idc.com/getdoc.jsp?containerld=prUS24298013 [accessed 12/11/2014]

[6] J. Martinez, IBM attempts to conquer the cloud with plans to introduce 20 new industry-specific tools, TechRadar Pro, available at: http://www.techradar.com/news/internet/cloud-services/cloud-and-clear-ibm-tointroduce-20-subscription-based-cloud-tools-1250879 [accessed 12/11/2014]

[7] IBM Press Release, IBM Expands Industry-Specific Cloud Consulting Services http://www03.ibm.com/press/us/en/pressrelease/42676.wss [accessed 12/11/2014]

[8] P. Kaushik, SAP Announces Industry Specific Cloud Roadmap, TJ Tools Journal, 2014, available at: http://www.toolsjournal.com/cloud-articles/item/3256-sap-announces-industryspecific-cloud-roadmap [accessed 09/11/2014] 
[9] J.Martinez, VMWare launches industry-specific remote desktop tools, TechRadar Pro, available at: http://www.techradar.com/news/internet/cloud-services/vmware-launches-industry-specificremote-desktop-tools-1255144_[accessed 12/11/2014]

[10] Legal IT professionals, dinCloud Announces Legal Industry Specific Cloud Services, available at: http://www.legalitprofessionals.com/usa-news/6447-cloud-law-firm-dincloud-launch [accessed $12 / 11 / 2014]$

[11] Cetrom, Cloud Computing by Industry, http://www.cetrom.net/cloud-computing/cloud-industrysolutions/_[accessed 12/11/2014]

[12] A. Sun, Enabling collaborative decision-making in watershed management using cloudcomputing services, Environmental Modelling \& Software 41 (2013) 93-97.

[13] M. Matthies, C. Giupponi, B. Ostendorf, Environmental decision support systems: current issues, methods and tools, Environmental Modelling \& Software, 22 (2007)123-127

[14] O. Poppe, B. Jolliffe, D. L.Adaletey, J. Braa, and A. S. Manya, Cloud Computing for Health Information in Africa? Comparing the Case of Ghana to Kenya, Health Informatics in Africa, 1 (2013)

[15] R. K. Perrons, and A. Hems, Cloud computing in the upstream oil \& gas industry: A proposed way forward, Energy Policy 56 (2013) 732-737.

[16] G. Dán, R. B. Bobba, G.Gross, and R. H. Campbell, Cloud Computing for the Power Grid: From Service Composition to Assured Clouds, in: Proceedings of the 5th USENIX Workshop on Hot Topics in Cloud Computing, USENIX Association, 2013.

[17] O. Wenge, U. Lampe, A. Müller, and R.Schaarschmidt, Data Privacy in Cloud Computing - An Empirical Study in the Financial Industry, in: Proceedings of the Twentieth Americas Conference on Information Systems, Association of Information Systems, 2014

[18] V. X.Wang, and X.W. Xu,An interoperable solution for Cloud manufacturing, Robotics and Computer-Integrated Manufacturing 29 (2013) 232-247.

[19] M Meier, J Seidelmann, I Mezgár, ManuCloud: the next-generation manufacturing as a service environment, ERCIM News, 83 (2010) 33-34

[20] R. Iqbal, A. James, and R. Gatward, A Framework for Integration of CSCW, in: Proceedings of the 7th International Conference on In Computer Supported Cooperative Work in Design, IEEE, 2002, pp. 43-48. 
[21] H. Panetto, and J. Cecil, Information systems for enterprise integration, interoperability and networking: theory and applications, Enterprise Information Systems 7 (2013): 1-6.

[22] N. Karacapilidis, N. S. Christodoulou, M.Tzagarakis, G.Tsiliki, and C. Pappis, Strengthening collaborative data analysis and decision making in web communities, in: Proceedings of the Companion Publication of the 23rd international conference on World Wide Web, 2014 pp. 10051010.

[23] P. Gupta, A. Seetharaman and J. R. Raj,The usage and adoption of cloud computing by small and medium businesses, International Journal of Information Management 33 (2013) 861-874.

[24] Q. Li, Z.Wang, W. Li, J. Li, C. Wang, and R. Du, Applications integration in a hybrid cloud computing environment: modelling and platform, Enterprise Information Systems 7 (2013): 237271.

[25] Z. Zhang, C. Wu, and D. WL Cheung, A survey on cloud interoperability: taxonomies, standards, and practice, ACM SIGMETRICS Performance Evaluation Review 40 (2013), 13-22

[26] A. Sill and G. Kecskemeti, Guest Editors' Introduction: Special Issue on Interoperability, Federation Frameworks and Application Programming Interfaces for laaS Clouds, Grid Computing 12 (2014) 1-2.

[27] D. Petcu, G. Macariu, S. Panica, and C. Crăciun, Portable cloud applications-from theory to practice, Future Generation Computer Systems 29 (2013), 1417-1430.

[28] I. Mezgár and U. Rauschecker, The challenge of networked enterprises for cloud computing interoperability, Computers in Industry 65 (2014) 657-674.

[29] S. Marston, Z. Li, S. Bandyopadhyay, J. Zhang, and A. Ghalsasi, Cloud computing - the business perspective, Decision Support Systems 51 (2011) 176-189.

[30] H. Demirkan and D. Delen, Leveraging the capabilities of service-oriented decision support systems: Putting analytics and big data in cloud Decision Support Systems 55 (2013) 412-421.

[31] Z. Ma, Y. Li, and F. Zhou, An e-commerce-oriented creditworthiness service, Service Oriented Computing and Applications (2014) 1-8.

[32] K.Chao, A. E. James, A. G. Nanos, J.Chen, S.Stan, I.Muntean, G.Figliolini, P. Rea, C.B. Bouzgarrou, P.Vitliemov, J. Cooper, J.van Capelle, Cloud E-learning for Mechatronics: CLEM, Future Generation Computer Systems, this issue. 
[33] N. Kumar, R. Iqbal, S. Misra, J. J.P.C. Rodrigues, Bayesian Coalition Game for Contention-Aware Reliable Data Forwarding in Vehicular Mobile Cloud, this issue.

[34] S.Ma, C.Lan, C.Li, Contextual Service Discovery Using Term Expansion and Binding Coverage Analysis, Future Generation Computer Systems, this issue.

[35] D. Shen, J. Luo, F. Dong, X. Fei, W. Wang, G. Jin, W. Li, Stochastic modeling of dynamic rightsizing for energy-efficiency in cloud data centers, Future Generation Computer Systems, this issue. 